\title{
Quantifying Workers' Hazard Identification Using Fuzzy Signal Detection Theory
}

\author{
Tariq Sami Adbelhamid*, ${ }^{*}$, Puneet Narang ${ }^{2}$ and Don W. Schafer ${ }^{3}$ \\ ${ }^{I}$ Michigan State University, School of Planning, Design and Construction, East Lansing, MI 48824-1323, USA \\ ${ }^{2} K P M G$, India \\ ${ }^{3}$ Building Science, Appalachian State University, Boone, NC 28608, USA
}

\begin{abstract}
Workers' safety and health is a primary concern in the construction industry due to the significant number of occupational injuries and fatalities experienced. Previous research indicates that such injuries and fatalities are multicausal, and one of which may be workers drifting towards hazard. The purpose of this study was to develop a method to quantify the ability of construction workers to identify hazards such that this drifting can be minimized. The paper is not reporting on a specific finding regarding hazard identification ability amongst the population of workers surveyed in the research. Rather, it is a foray into a new method that augments assessment of hazard identification abilities of workers. This research applied a hybrid model, Fuzzy Signal Detection Theory (FSDT) to quantify workers' ability to identify occupational hazards. Data was collected using a survey designed to assess construction workers' perceptions of safety specific to the risk of fall accidents for structural steel workers. An eighteen-question survey was based on standards set by national safety agencies in the United States and on a mix of conditions that were with no violation, a complete violation, and a partial violation. Both the conventional Signal Detection Theory (SDT) and FSDT were used for analysis and the results were compared. The comparison of results indicated that the FSDT model provided a richer framework to study worker hazard perception on a construction site. The methodology is expected to provide guiding framework for similar studies. Increasing data collection of the type presented in this paper will enable refinement and revision of construction occupational safety and health regulations by national agencies.
\end{abstract}

Keywords: Construction safety, construction hazard, hazard identification, signal detection theory.

\section{INTRODUCTION}

Each year, occupational injuries (traumatic, ergonomic, and/or exposure) and fatalities temporarily or permanently disable many and claim the lives of people in the construction industry. The staggering statistics collected and disseminated by occupational safety and health concerned organizations (NIOSH, U.S. Department of Labor, MIOSHA) confirm the impact and importance of this problem. With increased attention on safety, company-wide safety programs became the norm. Many employ full time safety officers or consultants to assure that legal requirements are met and, hazards and incidents are reduced. Safety programs create a counter pressure that aims to minimize exposure to hazards and keep workers away from hazardous situations. These programs are in place but the industry still remains dangerous. Recent research results indicate that the dynamic nature of construction work and the transient nature of the workforce make the prevention of accident scenarios difficult [1].

Further improvement is needed, but improvement has reached a plateau and construction still kills or injures more than eight percent of its workers each year, and claims $23 \%$

*Address correspondence to this author at 214 Human Ecology, School of Planning, Design and Construction, Michigan State University, East Lansing, MI 48824-1323, USA; Tel: 517-432-6188; Fax: 517-355-7711; E-mail: Tariq@msu.edu of all occupational fatalities and $10.5 \%$ of all occupational injuries $[2,3]$. Considering that the construction workforce accounts only for $6 \%$ of the US workforce, the disparity in the proportion of construction workforce and the accidents related to it is a clear indication of the serious problem in the construction industry.

To analyze construction worker safety on a job-site, it is important to understand the mechanics of safety implementation efforts. If we take any construction jobsite as an example, there is a myriad of conditions that could lead to accidents. Of course there is a gap between the accident actually occurring, and an existing condition, i.e., potential for an accident. How wide or narrow is this gap, depends upon how well the management and workers are prepared to handle a dangerous situation.

The first point of contact is inevitably the worker, who is performing his/her job and often needs to make a decision that would result in the potential condition transforming into a dangerous one and eventually into an accident. The worker's action simply would release the hazard, or create one for another worker. Consequently, hazard identification, or the lack thereof, by workers is a most critical aspect in successfully implementing safety regulations and guidelines. While not the primary focus of this research, other factors like adequate personal protection equipment, proper worker training with the means and methods, company policies and mandatory guidelines are also important, and are constantly evolving and form the foundation for all safety training. 
Teaching workers to recognize that they have stepped into a hazardous situation (zone) is typically achieved through intensified and directed training or training reinforcement such as signage. However, workers may not consider the same hazardous situation or risks in the same manner, because hazard recognition is contextual and subject to individual judgment and experience $[3,4]$. In other words, one worker may consider a situation hazardous while another worker would consider it perfectly safe. As a result, the sensitivity of workers towards unsafe conditions will be different and the tendency to work in known hazardous situations will depend on a worker's risk orientation.

This topic has received less attention compared to other factors such as personal protective equipment and mandatory guidelines and directives that are employed in the form of written instructions and formal training. This research proposes that assessment of hazard identification ability of workers is imperative in understanding the dynamics of construction accidents under different situations.

Based on the work of [2], which proposed that workers must be trained to recognize hazards and how to respond to them in a correct manner, [1] and [3] further explored the need for an assessment of the process of hazard identification. The application of Signal Detection Theory (SDT) was proposed for assessing construction workers occupational safety competencies. Although the application of SDT provided a good initial understanding of the hazard identification process in construction, the SDT model fails to capture all the information because of its intrinsic binary structure. In particular, SDT works on a yes/no (i.e. signal/noise) paradigm assuming it is possible to have two distinctively clear states.

This paper considers the use of fuzzy SDT, developed [4], as a means to expand the applicability of conventional SDT analysis to real world construction settings where the definition of a signal event is non-dichotomous. Basically, in fuzzy SDT, the binary definition of a signal is fuzzified by allowing an observer to assign non-binary membership degrees to a particular signal. Use of fuzzy SDT is justified given that real world situations are not always clearly defined, with every decision making task having uncertainties involved.

The specific aims of this research were as follows:

1. Introduce a fuzzy-based SDT method to assess a worker's ability to identify occupational hazards.

2. Demonstrate the fuzzy SDT method by designing and conducting a survey to assess construction workers' perception of safety, specific to the risk of fall accidents.

A brief description of prior research efforts in construction safety and SDT follows. Application of the proposed method is demonstrated using a study involving 30 structural steel workers. Results from the sample of 30 ironworkers are presented. A comparison between conventional SDT and fuzzy SDT and, reflection on the results concludes the paper.

This paper is not reporting on a specific finding regarding hazard identification ability amongst the population of workers surveyed in the research. Rather, this paper presents pre- liminary results of a new method that could possibly augment assessment of hazard identification abilities of workers.

\section{BACKGROUND}

\section{Construction Occupational Safety and Health}

Various parties have made efforts to reduce injuries and fatalities in construction and other work sectors by developing or implementing prevention plans and techniques [5-11]. These efforts have concentrated on managerial prevention plans, safety program implementation, and worker safety training [12-14].

To aid in identifying the underlying root causes behind accidents, construction accident causation models were proposed in the literature by a handful of researchers. McClay's 'universal framework' $[15,16]$ identifies three key elements of accidents: hazards, human actions, and functional limitations that are exceeded in the case of an accident. Hinze's distraction theory [17] argues that production pressures or other stress factors can distract workers from the hazards and increase the probability of accidents. The 'constraintsresponse' model [14] illustrates that any project condition or management decision (distal factors) can cause responses that create inappropriate conditions or actions (proximal factors) that may lead to accidents. The root cause analysis model by [18] identifies three general root causesmanagement deficiencies, training, and workers' attitude. [19], proposed eight root causes: lack of proper training, safety equipment not provided deficient enforcement of safety, unsafe equipment, method, or condition, poor safety attitude, and isolated deviation from prescribed behavior. It was proposed in [20] that an accident causality model (ConCA), should consider three influence hierarchies in construction accidents, namely, immediate accident circumstances, shaping factors, and originating influences.

Despitethe contributions of construction accident causation models to understanding the accident process, none adequately explain the underlying causes of construction accidents. The difficulty in fully explaining construction accidents may be attributable to the dynamic nature of construction work and the different ways in which accidents occur from site-to-site. Moreover, following preset rules can be applied in a well-structured environment where changes are predictable and controllable. But this is not possible in dynamic conditions and environments such as those characteristic of construction.

In [2], a new model was proposed for understanding construction accidents by providing a link between all the above-mentioned models and uniting them under Rasmussen's model. In his theory of "Cognitive System Engineering", Rasmussen argues that there are no objective stop rules for tracing the cause of events [21]. According to this theory, the analysis stops when an explanation makes sense from the analyst's perspective. He provides five different perspectives and the point at which each of them is satisfied, limiting the range of potential causes of the error/accident. An important conclusion from Rasmussen's model (Fig. 1) was that workers must be trained to recognize hazards, because only when they are able to identify safe and unsafe conditions, they would respond to perceived and actual risks in the correct manner. 


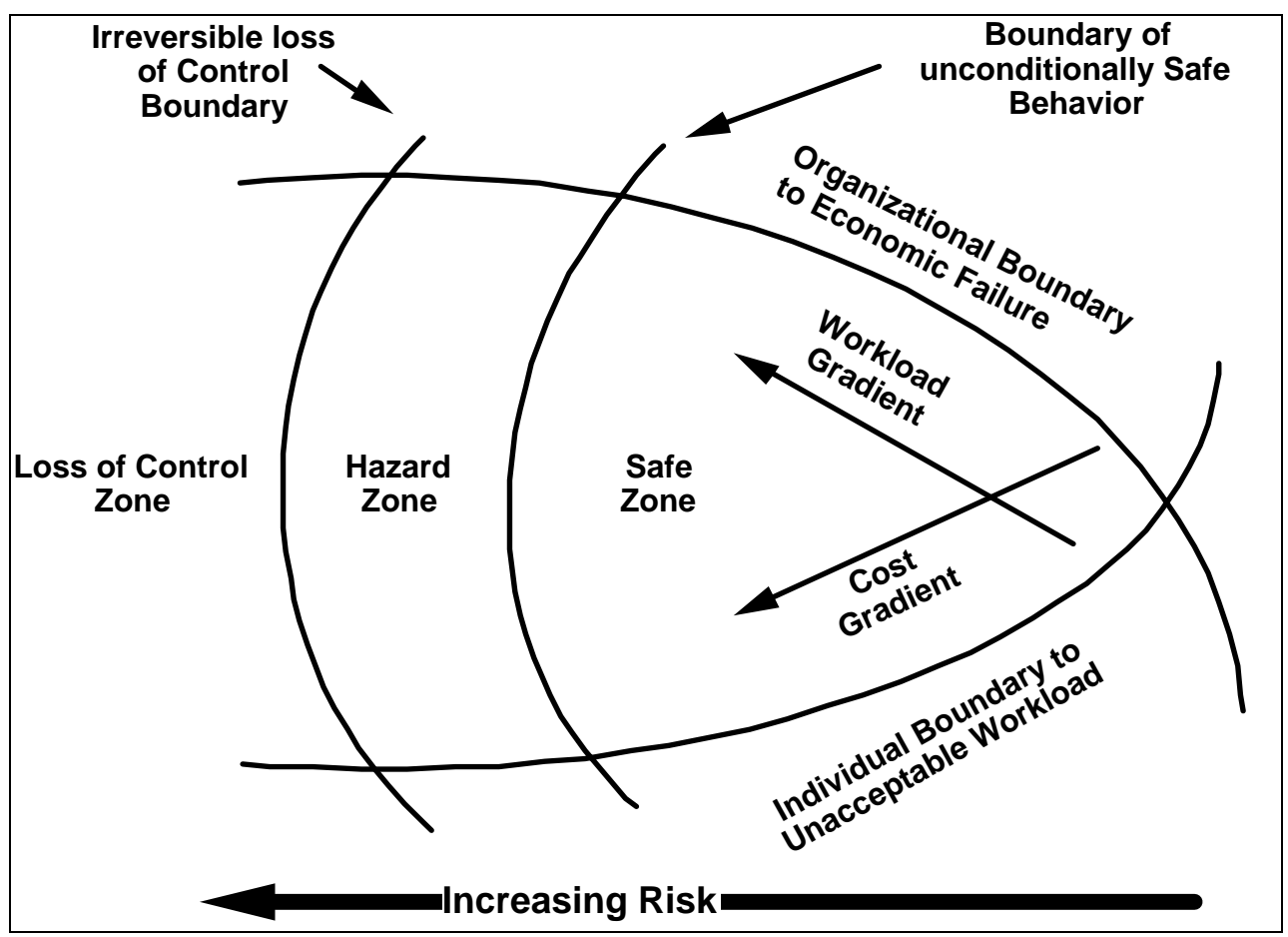

Fig. (1). Rasmussen's original model: Three zones of risk [2].

As shown in Fig. (1), the original Rasmussen's model, the work environment comprises three zones: Zone I or the safe zone, Zone II or the hazard zone, and Zone III or the loss of control zone.

Zone I or the safe zone is enclosed by the "Boundary of Unconditionally Safe Behavior", "Organizational Boundary to Economic Failure", and "Individual Boundary to Unacceptable Workload"[21, 22]. Zone II or the hazard zone is encompassed by the "Boundary of Unconditionally Safe Behavior" and the "Irreversible Loss of Control Boundary".

Rasmussen states that due to economic or workload pressures, workers will shift their work along the workload and/or cost gradients, respectively. Both gradients move from a high value to a low value. Thus, as long as workers remain within the safe zone, work activities can be safely performed. Current safety regulations and management practices are directed at keeping the workers in the safe zone. Rasmussen suggests that enlarging the safe zone through proper planning of operations will make the work safer.

Workers working in the Hazard zone (Zone II) are considered to be working at the edge. Hazard zone includes hazards that could result in traumatic, exposure, and/or ergonomic type injuries as well as fatalities.

Zone III in Rasmussen's model is the loss of control zone where accidents occur and control is lost leading to injuries and/or fatalities. Rasmussen proposes that workers should be educated and trained on how to recover from situations encompassed by the third zone. This is very similar to instructing drivers on how to handle slips on icy roads.

Rasmussen's theory recognizes that organizational and individual pressures will push people to work in hazardous situations. These pressures defeat efforts to enforce safe work rules specifically in a changing work environment such as in construction. Therefore, this approach emphasizes the need to train workers to be conscious of hazardous work environments and to engage the work with better planning and appropriate protection in a way very similar to how fire fighters engage hazardous situations. According to Rasmussen the worker is the best person to judge the boundaries of safe work. So instead of forcing workers to follow the rules and stay in the safe zone, Rasmussen suggested that workers be trained to:

1. Identify in which zone they are working.

2. Identify hazards.

3. Prevent hazard release.

4. Recover when hazards are released.

While counterintuitive, Rasmussen's recommendation to train workers to deal with hazards and recover from scenarios when control is lost recognizes that workers will frequently and inevitably work in the hazard zone. Management pressures and seeking less physical, and perhaps mental, workload effort are realistic examples of what may push workers to the hazard zone. While Rasmussen still maintains that enlarging the safe zone will increase safety and performance, he acknowledges that there is need to tell workers what to do in the 'hazard' zone and when control is lost.

Stated differently, the Rasmussen model is basically advocating a similar approach to social norms marketing used by social scientists. Proponents of this approach maintain that informing people 'what to do' instead of the traditional approach of telling people 'what not to do' is a better way of achieving desired behavioural changes. For example, instructing college-aged adults who are also heavy drinkers not to drink before driving and citing the grave consequences of such behavior has become an overrated message that lost its effectiveness. Alternatively, social marketing follows a tact 
where people are given instruction on what to do if they would like to drink - to drink moderately and have fun while sparing yourself and others the risks.

The acceptance and effectiveness of Rasmussen's approach remains an open question that only future research can answer. In [2], it was recommended that future research efforts consider the following three areas:

1. IN THE SAFE ZONE: Establish methods and techniques to enlarge the safe zone.

2. AT THE EDGE: Train workers on the identification of safe and unsafe conditions. And once in an unsafe condition, workers should be trained on how to recover from errors.

3. OVER THE EDGE: People will inevitably make mistakes resulting in loss of control. Hence, measures should be in place to limit the effect of this loss.

Of these three areas, this paper is concerned with the ability of workers to identify safe and unsafe conditions (Zone II - At the Edge). In [1] and [3], the application of Signal Detection Theory (SDT) for assessing a construction worker's ability to identify occupational hazards was explored. However, the SDT model fails to capture the subtlety behind a real work situation because of its intrinsic binary structure (i.e. signal vs. noise). Conventional (crisp) SDT works on a yes/no paradigm. Construction, like many other industries, is a real world setting; which is not devoid of its share of uncertainties. To provide an accurate assessment of construction safety competencies, this paper investigates improving the SDT-based model using a Fuzzy SDT approach.

\section{From Crisp Signals/Responses to Fuzzy Signals/ Responses}

Almost all reasoning and decision-making takes place in the presence of some uncertainty. A certain level of compe- tency is required in the given area to make the correct decision. There is always a relationship between physical stimuli, sensory response, and our ability to detect the two. Signal Detection Theory (SDT) provides a basis to measure this relationship. SDT is a method of assessing a decision making process when a person or a machine has to detect or recognize stimuli and respond accordingly. SDT is a welldeveloped psychophysical approach to quantify the ability of an observer to discern between signal and noise, where signal (s), is a state/stimulus in which the event of interest is present and, noise (n), is a state/stimulus in which the event of interest is absent $[23,24]$.

Two important parameters of the SDT analysis are: "Sensitivity" (d') and "Bias" (B $\beta)$ of the observer. Sensitivity refers to how well a person can discriminate between whether a signal is present or absent, and response bias refers to the inclination of a person to agree with the presence or absence of a signal. These two together explain how an operator would detect a stimulus and respond.

\section{RESEARCH METHOD}

Despite all the functions and usefulness of the conventional SDT, it fails to capture a certain amount of information due to its binary nature. Therefore, this study used Fuzzy SDT, to expand the applicability of conventional SDT analysis to real world construction settings by application of fuzzy logic to responses [25]. As compared to conventional SDT, where the responses, 'yes' and 'no' and are represented as 1 and 0 respectively; in FSDT, 'yes' and 'no' are represented to the extent it is meant by the respondent, which could be any value between 0 and 1 .

\section{Signal Detection Theory}

As shown in Table 1, in a conventional SDT model, the worker would respond in a binary manner, either saying yes $=1$, hazard or signal present, and would stop work $=0$ or would respond no $=0$ (signal absent) and carry on work $=1$.

Table 1. Possible Outcomes of Crisp SDT.

\begin{tabular}{|c|c|c|c|c|c|c|}
\hline Signal 's' & Response'r' & Hit & False Alarm & Miss & Correct Rejection & $\begin{array}{c}\sum \\
\text { H+FA+M+CR }\end{array}$ \\
\hline \hline 0 & 0 & 0 & 0 & 0 & 1 & 1 \\
\hline 1 & 0 & 0 & 0 & 1 & 0 & 0 \\
\hline 0 & 1 & 0 & 1 & 0 & 0 \\
\hline 1 & 1 & 1 & 0 & 1 \\
\hline
\end{tabular}

Table 2. Possible Outcomes of Fuzzy SDT

\begin{tabular}{|c|c|c|c|c|c|c|}
\hline 's' & ' $\mathbf{r}$ ' & $\mathbf{H}$ & FA & $\mathbf{M}$ & CR & $\begin{array}{c}\sum \\
\mathbf{H}+\mathbf{F A}+\mathbf{M}+\mathbf{C R}\end{array}$ \\
\hline 0.8 & 0.9 & 0.8 & 0.1 & 0 & 0.1 & 1 \\
\hline 0.2 & 0.2 & 0.2 & 0 & 0 & 0.8 & 1 \\
\hline 0.5 & 0.2 & 0.2 & 0 & 0.3 & 0.5 & 1 \\
\hline
\end{tabular}


Both the signal and the response only take binary values. Even if the worker is uncertain, there is always a binary response generated. Table 1, provides a truth table for conventional SDT, wherein for all possible conditions the worker would yield a value that would populate only one out of the four outcomes, and the rest would have zero membership.

\section{Mechanics of Fuzzy Signal Detection}

Fuzzy SDT, on the other hand, as shown in Table 2, recognizes that the worker response is subject to an overlapping membership in the two sets of 'yes' and 'no'. Not all responses are distinctively clear between two states, just like not all stimuli are distinctively clear between two states. There is a degree to which an event is a signal, i.e., an unsafe condition, and a corresponding degree (for the same event) to which it is a safe condition. Accordingly, there is a degree to which a signal present response (or, yes this is an unsafe condition) is made, and a smaller degree to which the same response would be no, signal is absent.

Similar to conventional SDT, concepts of "Hit" (correct signal detection), "Miss" (failed to detect a signal present), "False Alarm" (detected a signal when none present) and "Correct Rejection" (decided no signal present when none present) are valid in the FSDT; it is their binary characteristic that is discarded because of the loss of valuable information. Hence, each event represented by a stimuli-response pair in FSDT belongs, with some degree, to more than one of the four categories used in conventional (or crisp) SDT. Consequently, it is possible that events would claim nonzero membership in more than one outcome category, as shown in Table 2 (' $\mathrm{s}$ '= degree to which an event is a signal; varies from 0 to 1 ; ' $r$ '= degree to which a 'yes' (signal present) response was made; varies from 0 to 1 ).

\section{APPLICATION OF FUZZY SIGNAL DETECTION}

In order to apply FSDT to a construction jobsite setting, it is important to understand the worker's perception, which is therefore transformed into quantifiable data. The SDT literature, $[1,2]$ indicates that when a worker is faced with a hazardous situation, he or she mentally perceives the strength of the stimuli. Therefore, the first step would be to map the stimuli according to variables that describe the state of the world into the signal set - 's', with some degree of membership varying from zero to one.

In the context of a construction site, the defining variables are severity of the state of world that inclines towards an unsafe condition. For example, some common stimuligenerating situations that a worker faces are: an unprotected or exposed drop of more than six feet, a protruding rebar without a protective cap, a crane operation in close proximity to power lines, and a faulty steel connection. The variable in each of these situations would be the severity of the situation. Each situation that the worker is faced with is equivalent to a mapping between 0 and 1 , but there is no such single variable that could be globally used to convert a signal strength to an's' value. This mapping is given by the following equation (1): $[\mathrm{s}(\mathrm{SW})=\mathrm{f}(\mathrm{x})]$. In equation (1), the value of ' $s$ ' is calculated as a function of the severity of unsafe condition. Since the severity does not depend upon any one single on-site variable, it is not possible to propose an overall function that includes all possible unsafe conditions on construction sites.
The proposed method to measure in this research was a linear scale ranging from $0=$ least severe to $10=$ most severe. For example, a 'zero' would be a condition with a very low likelihood of hazard release and, a 'ten' would be a condition with a high likelihood of leading to a fatality. Labeling of a condition with a zero-rating as a "safe condition" was avoided because it would be almost impossible to prove any condition "absolutely harmless" on a typical construction job-site. However, discussion with safety managers and workers indicated that it would be more appropriate to rate a condition zero to describe it as "absolutely safe". This does provide a worker with a range that has two clear extremes, and hence, aids in generating a more realistic response to the presented stimuli. The intermediate values were only whole numbers. The continuity of the evidence variable was not sacrificed and the range from 0 to 10 helped collate the worker's hazard detection ability. To transform this into an 's' value, i.e., to determine its membership in the signal set, a factor of 0.1 was used.

The response generated by the worker was mapped to the ' $r$ ' set with a membership ranging from 0 to 1 . The response value (RV) is dependent upon the worker's conviction in giving either responses, namely, 'yes - signal present' or 'no-signal not present'. The sole variable here is the level of conviction, given by equation (2) as: $[\mathrm{r}(\mathrm{RV})=\mathrm{f}(\mathrm{y})]$. In equation (2), the value of ' $r$ ' is calculated as a function of the worker's level of conviction. For the fuzzy SDT model to perform effectively, it is desirable to define both ' $s$ ' and ' $r$ ' on similar (if not identical) scales, hence a linear scale similar to 's' was used that would produce the variable value ranging from 0 to 10 . The factor used for transforming the response to its r-membership is also 0.1 .

\section{Data Collection}

The ideal method to determine and analyze worker responses would require real time assessment on a construction job-site. The worker, when faced with both states, safe and unsafe, would make a decision based on his/her understanding of the physical context and his/her analysis of the risk involved. Though this approach would yield data in its native state, it is highly dangerous and practically not feasible. Hence the alternative method adopted is a survey that represents various site conditions pictorially.

Based on the safety standards set by agencies like OSHA (Occupational Safety and Health Administration), NIOSH (National Institute for Occupational Safety and Health) and ANSI (American National Standards Institute), there were potential conditions selected that could result in fall accidents. There were other safe conditions also selected to balance the overall survey and provide the subject with a realistic mix of questions. It was very important to present these scenarios, as close as possible to how a worker encounters them on site. In order to achieve this, the conditions were further short-listed to only retain those that could be presented visually, in the form of real photographs. This would enable the worker to select the most natural answer and capture a truer hazard identification ability of the worker.

The subject would select a value for the two questions on each of the eighteen conditions, resulting in the ' $s$ ' and ' $r$ ' pair. Each worker according to his/her understanding first selected a value based on how safe he/she thought the pre- 
sented condition was. This value is multiplied by 0.1 to yield the membership in the signal set, i.e., 's' value. Similarly, the second question (how sure is the worker) calls for a response from 0 to 10 , which results in the ' $r$ ' value, i.e., membership in the 'Yes-signal present' set.

The following example will illustrate how the values of ' $s$ ' and ' $\mathrm{r}$ ' are determined. In this example, a worker is presented with a situation as shown in Fig. (2). In the survey, the condition was specifically explained above the image, which showed that the ironworker was connecting fourth floor primary beams without a protected edge and decking in place on the lower floors and, that the ironworker was wearing fall protection (fall arrest) gear. The worker presented with this situation was asked two questions and was required to select one answer from the eleven possible values under each question.

The first question, namely, "How safe is this?" inquired about the strength of the signal, where the worker had to tell the two states apart - noise and signal. By expressing how safe the situation is, the worker provided a value for calculat- ing the sensitivity or the discriminability with which he/she had identified the hazard.

The second question inquired, "How sure are you about your answer?" The response to this question indicated the level of conviction with which the worker had answered the first question. An integer value ranging from 0 to 10 , where 0 was equivalent to "completely uncertain" and 10 was equivalent to "absolutely sure". The specific value selected reflected the response criterion of the worker towards the situation, indicating if the worker had used a risky or conservative strategy in making his decision about the safety of the presented situation (see [3] and [26] for interpretation rules and assumptions).

Assuming that a worker selects 7 for the first question and 6 for the second one, using equations 1 and 2, the values for ' $\mathrm{s}$ ' and ' $\mathrm{r}$ ' are 0.7 and 0.6 , respectively. The $\mathrm{s}$ and $\mathrm{r}$ pair would yield the following truth table for the four outcomes, as calculated using: $\mathrm{Hit}$ : $\mathrm{H}=\min (\mathrm{s}, \mathrm{r})$; Miss: $\mathrm{M}=\max (\mathrm{s}-$ $\mathrm{r}, 0)$; False Alarm: $\mathrm{FA}=\max (\mathrm{r}-\mathrm{s}, 0)$; Correct Rejection: $\mathrm{CR}=\min (1-\mathrm{s}, 1-\mathrm{r})$. The results are listed in Table 3.

6. An ironworker connecting $4^{\text {th }}$ floor beams, with an unprotected edge with no decking in place on the lower floors, protected by conventional fall protection (fall arrest).
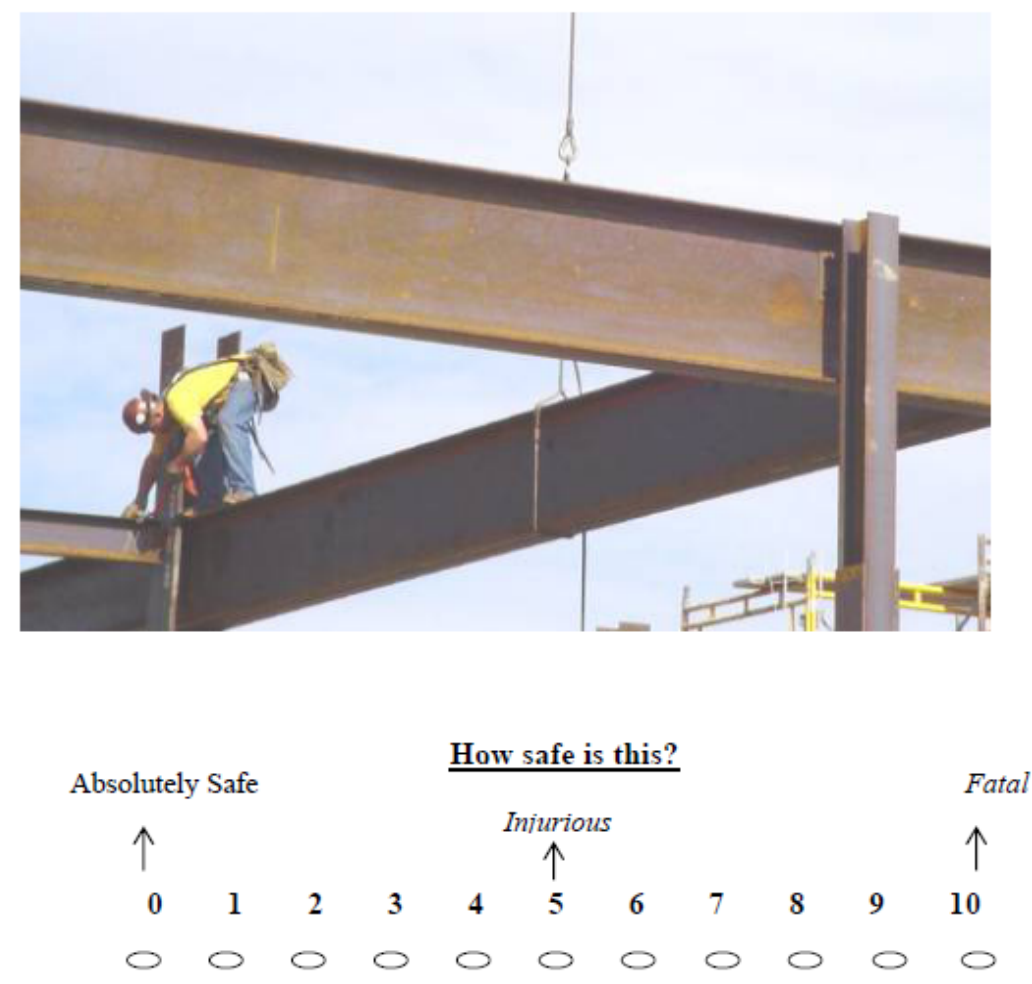

How confident are you about your answer?

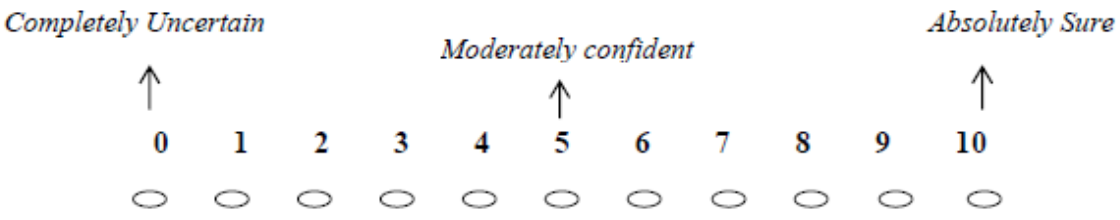

Fig. (2). Example to illustrate proposed methodology: Ironworker making initial connections. 
Table 3. Example to Illustrate Proposed Data Analysis Method

\begin{tabular}{|c|c|c|c|c|c|c|}
\hline $\mathbf{S}$ & $\mathbf{r}$ & $\mathbf{H}$ & $\mathbf{F A}$ & $\mathbf{M}$ & $\mathbf{C R}$ & $\begin{array}{c}\sum \\
\text { H+FA+M+CR }\end{array}$ \\
\hline \hline 0.7 & 0.6 & 0.6 & 0 & 0.1 & 0.3 & 1 \\
\hline
\end{tabular}

The varying degrees of membership under the four possible outcomes, and a cumulative score from a set of such questions, are then used to calculate the values for the two primary parameters, sensitivity (d') and response criterion $(\beta)$.

Sensitivity (d') measures how well a person can discriminate between whether a signal is present or absent. This is represented by the difference between the means of the signal and noise distributions. The inclination or bias of a person to say 'Signal Present' in response to a stimulus is captured by the response criterion $(\beta)$. These two parameters, $\mathrm{d}$ ' and $\beta$, are not connected to ' $\mathrm{s}$ ' or ' $\mathrm{r}$, but are indirectly affected by their values.

Table 4 gives a sample list of a twelve question survey with one worker's responses, providing an 's-r' pair for each question. The calculations for arriving at the two parameters, $\mathrm{d}^{\prime}$ and $\beta$ are also shown (the detailed equations are found in [26]). The average d' sensitivity or the discriminability index of each worker can be understood as the extent of separation between the two states (bell curves) of signal and noise. In Signal Detection Theory, a HR (hit rate)-FAR (false alarm rate) pair of $0.99-0.01$, yields an effective ceiling for sensitivity, where d' $=4.65$. An average performance would result in $\mathrm{d}^{\prime}=1.00$.

\section{RESULTS}

The research focused on ironworkers and falls, as a demonstration example, due to the high risk of accidents. The survey presented eighteen on-site conditions that an ironworker would typically be faced with during the steel

Table 4. Sample Calculations Based on Worker Response (s-r Pair)

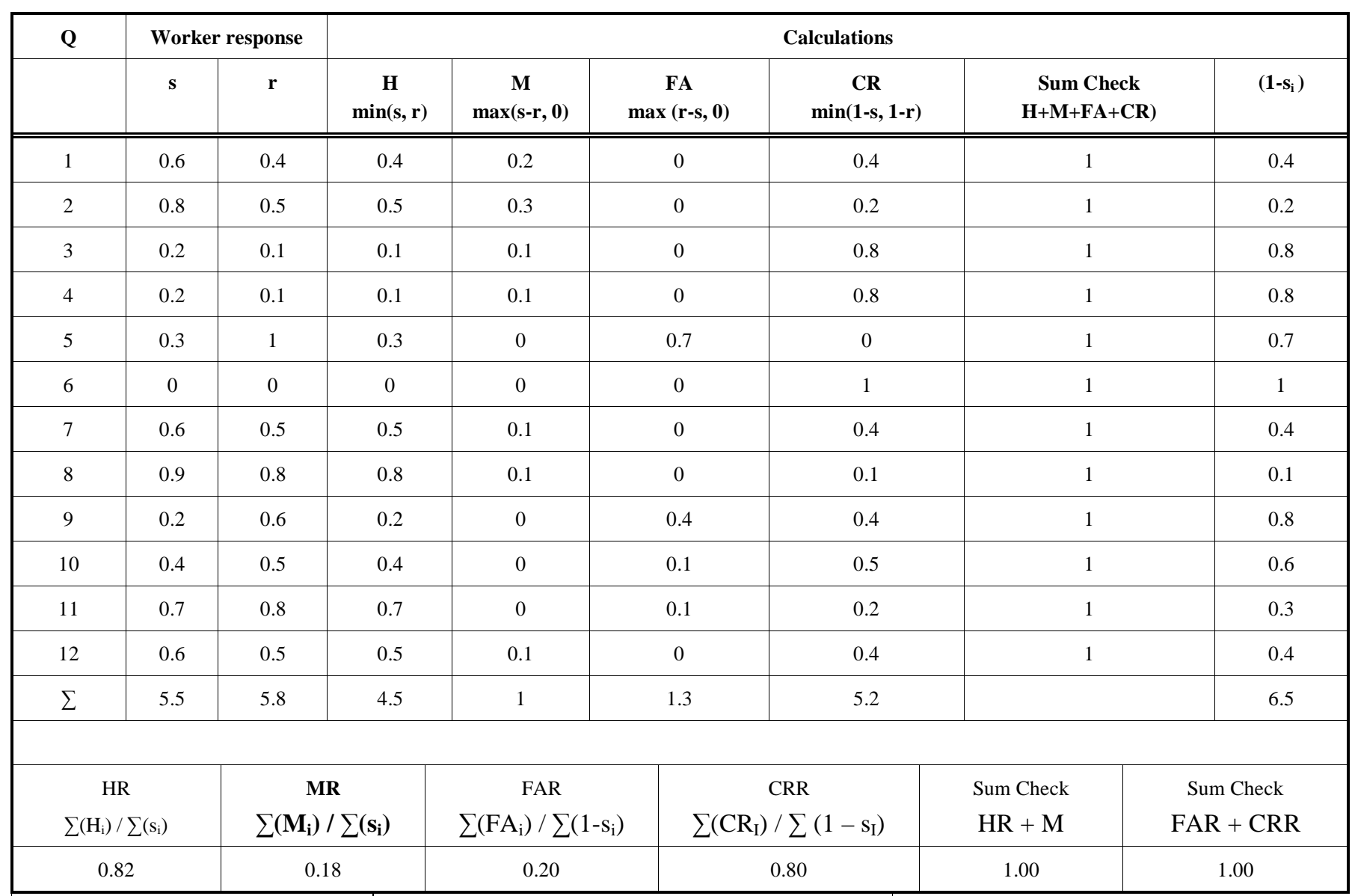

\begin{tabular}{|l|l|}
\hline$d^{\prime}=z(H R)-z(F A R)$ & $B=Y(H R) / Y(F A R)$ \\
\hline 1.76 & 0.9319 \\
\hline$H R=\sum\left(H_{i}\right) / \sum\left(s_{i}\right)$ for $\mathrm{i}=1$ to $\mathrm{N}$ & $\mathrm{MR}=\sum\left(\mathrm{M}_{\mathrm{i}}\right) / \sum\left(\mathrm{s}_{\mathrm{i}}\right)$ for $\mathrm{i}=1$ to $\mathrm{N}$ \\
$\mathrm{FAR}=\sum\left(\mathrm{FA}_{\mathrm{i}}\right) / \sum(1-\mathrm{si})$ for $\mathrm{i}=1$ to $\mathrm{N}$ & $\mathrm{CRR}=\sum\left(\mathrm{CR}_{\mathrm{i}}\right) / \sum\left(1-\mathrm{s}_{\mathrm{i}}\right)$ for $\mathrm{i}=1$ to $\mathrm{N}$ \\
\hline
\end{tabular}


Table 5. Calculation for Sensitivity ( $\left.d^{\prime}\right)$ and Response Criterion ( $\beta$ ) for all 30 Workers

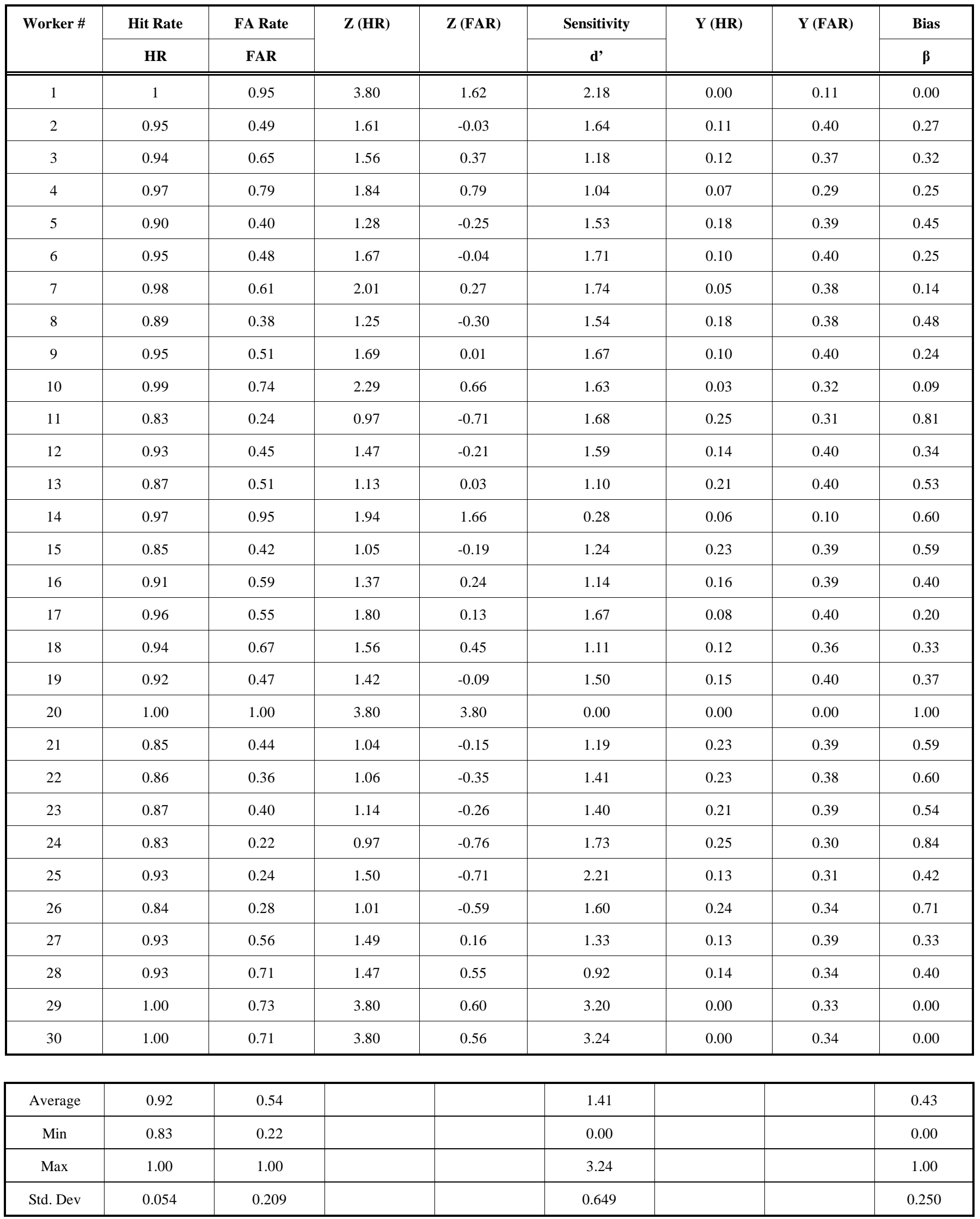


erection phase of any project. Because the survey already targeted a specific group (ironworkers), there was no attempt at further reducing the sample. All ironworkers were randomly included, with no restriction on age, gender, or years of experience in the industry. The population surveyed comprised of randomly selected ironworkers whose average age was 35 and average construction experience was six years. All ironworkers were familiar with safety standards and had undergone some form of safety training, pertaining to their company policies. Therefore, it was expected that they had sufficient understanding of the conditions presented. Thirty complete surveys were received, which was reasonably large to allow the use of normal distribution to analyze the data. The survey can be found in [26].

The following pages present the survey results, with the basic calculations. The results of the 30 workers using fuzzy SDT is shown in Table 5. Analysis of the survey data is shown in Table 5, which indicated a high variation in the response to the first question - 'how safe is this condition?' This explains a variation in the manner each of the 30 subjects perceived the 18 conditions presented to them in the survey. Nonetheless the subjects were confident of their responses, which was confirmed by the higher mean for the responses to the second question - 'how sure are you about your answer?'

As shown in Table 5, the average d' (sensitivity) value for this group of ironworkers was found to be 1.41 (standard deviation $= \pm 0.649$ ), which based on SDT literature [27], shows an above average sensitivity to differentiate and detect an unsafe condition from a safe one. There are only three workers (\#14, 20 and 28) whose d' value falls below 1.00.

In addition to analyzing the group of the 30 workers as a whole, it is instructive to consider the results of a single ironworker. Worker \#17 is selected (at random) for further analysis. This worker scored a HR and FAR pair of 0.96 and 0.55 respectively, based on the mapping of ' $s$ ' and ' $r$ ' values. These can be better understood with the help of Fig. (3).

For worker's 17 sensitivity (separation between the two bell curves), there is a high Hit Rate recorded, but the False Alarm Rate is also very high, indicative of the conservative strategy adopted by the worker yielding a very low 'response criterion' value (the vertical divide). The conservative bias pulls the divide towards the noise curve and explains the high FAR. In the same manner a distribution could be drawn out for each of the 30 workers that would provide information as to how well they are able to discriminate between the two states (how sensitive they are) and what strategy they adopt (risky or conservative).

The decision-making strategy of the workers is reflected by the response criterion or the bias of the group. As shown in Table 5, the average response criterion measure $\beta$ for this group of 30 workers was 0.43 (standard deviation $= \pm 0.25$ ). This indicates that the group had a conservative strategy, which means they tended to have a high false alarm rate, i.e., consider some of the safe conditions as unsafe. Although the workers produce a high hit rate, they also produce a high number of false alarms.

In construction, a conservative strategy is definitely better as compared to a risky one, since a risky strategy would result in high misses, which translates to high accident costs, productivity loss and lower morale. With the proposed fuzzy SDT approach and gathered data using a well-defined survey, the resulting analysis is an accurate reflection of the way these ironworkers perceive safety on site (see [3] and [26] for interpretation rules and assumptions).

The fundamental principle of fuzzy SDT is the nonbinary characteristic of signal and response. The ironworkers' ability to identify a signal is mapped in the two-step process, represented by the two questions with each condition. The 18 conditions presented were a good mix of:

- Absolutely safe conditions, conforming to standards.

- Conditions that were clear violations of all safety standards and would lead to a serious accident.

- Conditions that violated safety standard but were not so clearly identifiable. These could result in injuries, minor and/or major.

For example, question \# 3 from the survey presents a condition where the distance between beams tied on a multiple rigging assembly for erection is 6 feet. The required standard separation between beams in a multiple rigging assembly as set by OSHA is 7 feet. This standard is based on

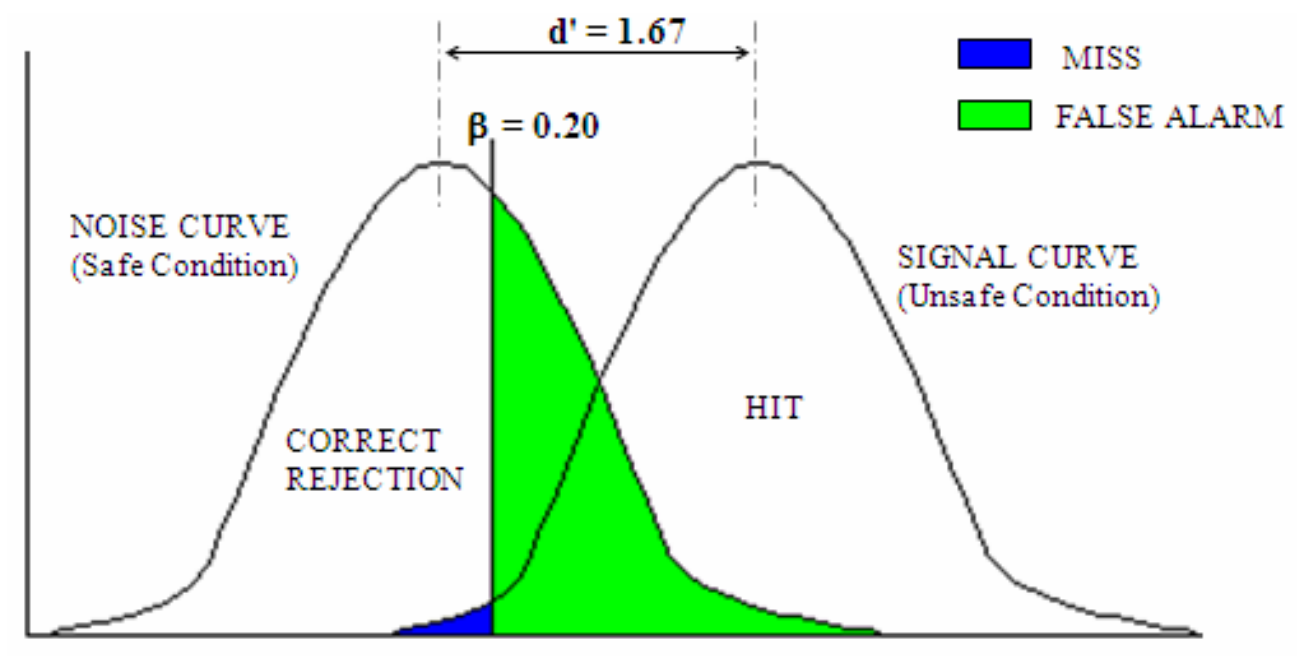

Fig. (3). Distribution of noise and signal for Worker \#17. 


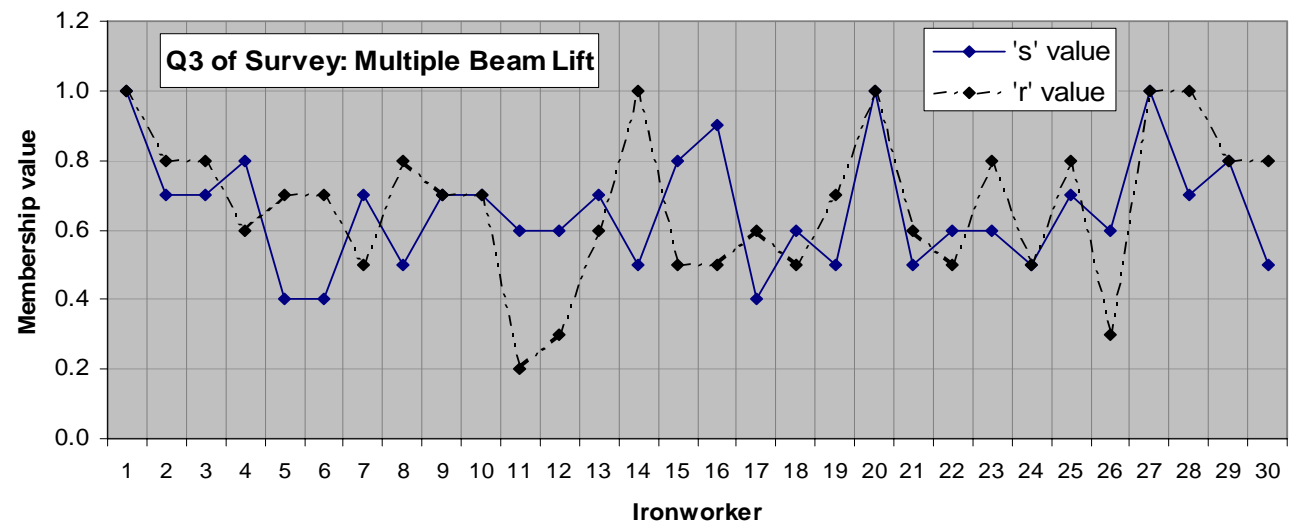

Fig. (4). 's' and 'r' responses to Q\# 3 by all workers.

the fact that when an ironworker is standing on a beam and the crane lowers the assembly, the 7 feet distance would avoid getting the worker hit on the head (when standing upright) as the worker lowers the first beam into position. So, this is potentially a dangerous condition presented to the ironworkers, but depending upon how tall each worker was, their perception of threat could vary. The degree of danger perceived by the worker, assuming they are aware of the standard from training, is captured by the second question. Fig. (4) presents a break up of individual responses to this condition. More details can be found in [26].

\section{DISCUSSION}

\section{Comparing Crisp to Fuzzy SDT}

To facilitate the comparison of conventional and fuzzy SDT, the workers' responses were analyzed using conventional SDT. Analysis of d' using conventional SDT resulted in a mean of 1.97 with standard deviation of \pm 1.10 . This is higher than the sensitivity found using fuzzy SDT, and with a noticeably higher standard deviation as well. Similarly, the measure of response criterion using conventional SDT was 0.61 on average with a standard deviation of \pm 0.3 . Fig. (5), which show a comparison of $\mathrm{d}$ ' and the response criterion using FSDT with the same parameters when calculated with conventional SDT. The figures clearly show that the sensitivity and response criterion derived using conventional SDT shows a sharp increase in the spread for the 30 subjects compared to same parameters derived from fuzzy SDT.

The preceding analysis indicates that the data analysis using Fuzzy SDT differs significantly from that based on conventional SDT. The significance of the difference in values obtained for either parameters $\mathrm{d}^{\prime}$ and response criterion lies in the final characterization of a worker's ability to identify hazards. For example, a worker may be regarded as having high sensitivity and conservative response criterion based on conventional SDT while fuzzy SDT could show him/her with a low sensitivity and risky response criterion. The wrong assessment may lead to an unnecessary course of action, such as less (more) training.

While the pilot sample size used is quite small, it is still possible to infer that for the same subject, sensitivity and response criterion values are very different between the two methods. The FSDT model clearly captures the workers sensitivity and response criterion (in the form of s-r pairs). The s-r pair then leads to the HR and FAR which on further statistical treatment result in the $\mathrm{d}^{\prime}$ and $\beta$ values. In crisp SDT, the binary setup forces a worker's response to the same questions into a single outcome set (H, M, FA, and CR). It is dur-
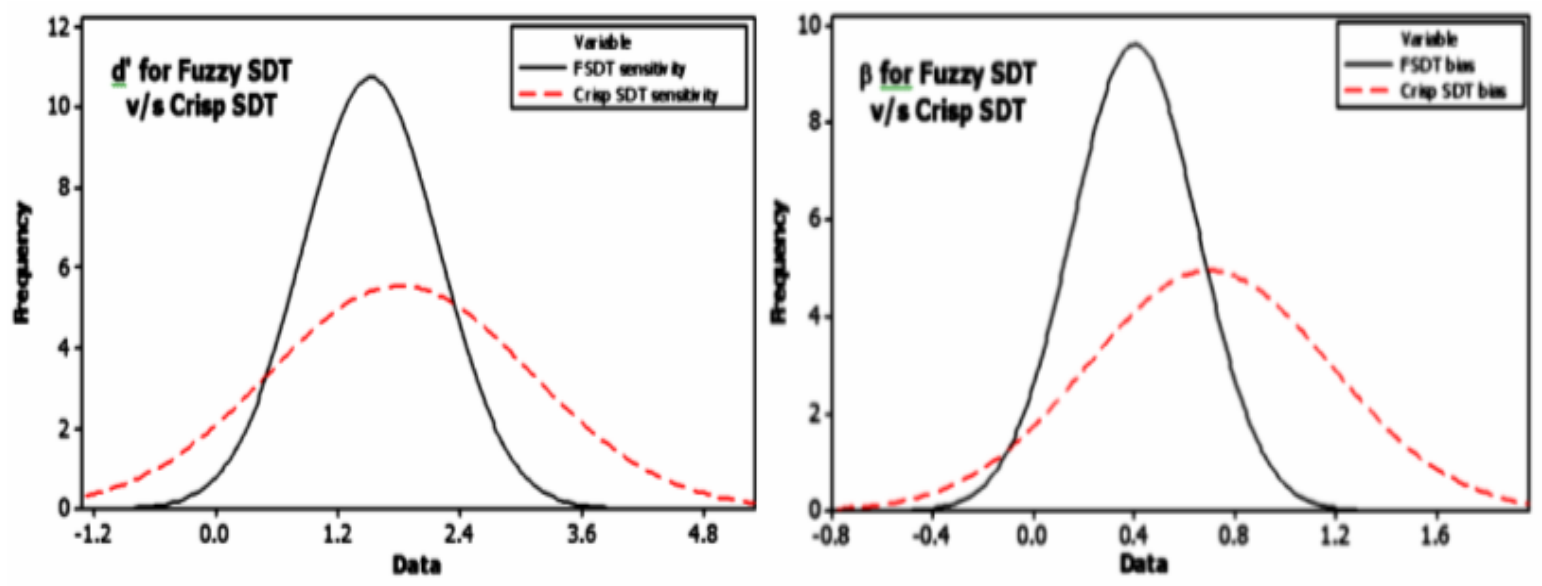

Fig. (5). Sensitivity (d') distributions for Fuzzy SDT $v / s$ Crisp SDT (left); Response criterion distributions for Fuzzy SDT $v / s$ Crisp SDT (right). 
ing this forced classification that the workers' ideal measure of their ability to sense a signal is confounded.

\section{Expert Analysis}

Four construction professionals were interviewed from the steel industry in mid and southeast Michigan:

- $\quad$ Engineering and Safety Manager for a leading steel fabrication corporation.

- $\quad$ Steel Erection Foreman (2) from two different steel erection subcontractors.

- $\quad$ Safety Director at a job site with a leading general contracting company.

The names of these individuals and companies are not disclosed for confidentiality. The discussions were based on the survey questionnaire. An important observation from these discussions was that an average ironworker on any jobsite is debriefed before the start of any project and updated with the most current safety regulations (OSHA, MIOSHA). Each project has its own safety and emergency plan drawn out and thoroughly discussed with all foremen, at the start of the project (or when that trade starts on the project).

There was a detailed discussion on the 18 conditions presented in the questionnaire and the following comments were received:

- $\quad$ Question \#3 pertaining to multiple lift rigging procedure for erecting beams requires the separation distance between two beams to be 7 feet. This is not always practiced on site, though if noticed by a safety professional it would account to a violation. The height of the crewmembers actually placing the beam in place is the critical dimension, and it is common these beams are separated by only 5.5 to 6 feet. This is an acceptable practice amongst the erection crew. They said they don't expect someone to stand between two rigged beams so they don't pay attention to the 7 feet separation, albeit they are aware of it.

- Though question \#6 (see picture in Fig. 1) presents an unsafe condition as per codes, the erection crew, if completely tied off and fastened by a fall arrest, did not consider working on an unprotected edge with a drop of more than 3 floors to be unsafe.

- Question \#8 presents a condition where a beam is connected with a single bolt at each end and the choker is released (it is detailed ${ }^{1}$ in much later). The codes clearly refer to this as a violation. This regulation has been in effect for over 35 years and some designers are believed to have developed a particular detail whereby this would be a completely safe practice. It has not yet been accepted by the safety agencies, but there is considerable momentum, that predicts a change is about to happen.

- $\quad$ Question \#14 presents a condition whereby only three out of the four anchor rods for a steel column are tightened off with nuts. This again is a violation of

\footnotetext{
${ }^{1}$ 'Detailing' in steel erection refers to the process of putting in the remaining nuts and bolts, and tightening the joint up. When the steel piece is set for the first time, it is not completely detailed (only part nuts/bolts are put in place). This process allows for the erection crew to make minor adjustments to plumb the structure up.
}

the codes, but a number of industry professionals accept the fact that on the job site, if there is one anchor rod damaged, many a times the column is still erected. They return to the fourth rod and repair it later.

All these scenarios are an indication of a gap in the safety regulations/standards and prevalent industry practices for steel erection. These are subtleties and finer nuances of the specialty trade that on numerous occasions transform into a potentially dangerous situation. Often the ironworker identifies this mismatch, but based on experience and expertise makes the decision of carrying on with work. There is an urgent need to bridge these gaps and make safety training more meaningful and effective. The involvement of actual ironworkers and their foreman, a discussion/dialogue between those who define these codes and those who these codes are designed to protect, could be the first step in this direction.

Another useful outcome of this study is that certain trends begin to appear in how workers are responding to a certain condition. For example, the majority of the workers (24 out of 30) considered working on a scaffold 10 feet above the lower level with no fall protection as safe, while it is in fact unsafe per regulations. Moreover, a majority of the workers falsely considered some safe conditions as unsafe. Understanding the dynamics behind such findings will be invaluable for development of worker-specific training and revisions of safe work rules.

The study results illustrate how the sensitivity and risk orientation of a worker can be determined. This information sets a benchmark against which the effectiveness of new training can be assessed. Essentially, this information would make it possible to determine if a worker's sensitivity and risk orientation towards safe and unsafe conditions increased, decreased, or remained unchanged as a result of the training. The use of fuzzy SDT proves to be a reasonable and accurate method to measure a worker's ability to judge the boundary beyond which work is no longer safe.

\section{RESEARCH LIMITATIONS}

This study covered only those aspects of fuzzy signal detection that would have immediate application for the purposes of identification of occupational hazard in the construction industry. For example, the definition of a signal and the generated response could have temporal and contextual variability, which implies dependence of signal definition on situation specific factors and variation of signal strength over time [4].

The survey methodology developed for this research has its own limitations. Once the surveys were distributed to the subjects there is no definitive way of concluding that they would behave in the same manner as they perform on the survey, if they were exposed to the actual condition. The survey questionnaire attempted to put as much detail, in terms of explaining the physical on site condition, with the help of photographs and text. In a few questions the visuals do not represent the question in its entirety, or may seem to fall short of the condition as explained by the text. The visual accompanying the text question must be an accurate explanation, leaving no room for the subject to make his/her own assumptions. 
A myriad of factors influence worker hazard identification, such as workgroup climate, experience type and length, etc. The determinants of worker hazard detection are not shaped by the attendance of a training session. The paper was concerned with the point in time where the worker faces a hazard and whether he/she is able to detect it. The schema the worker goes through to reach that decision, i.e., why the worker considered a situation as safe or not, was out of the scope of the paper.

Potential biases introduced by the survey/administrator, potential biases that would exist in the sample population, methods to ensure internal, external, and construct validity, and potential implications on the reliability of the results were not discussed in this paper. This is mainly because the paper is not intended to add a survey to the knowledge base but rather explore how fuzzy signal detection theory can be adapted to better quantify hazard identification in highly subjective situations.

\section{FUTURE RESEARCH DIRECTIONS}

Based on the current research findings and limitations, future research problems to consider are: comparison between the outcomes of crisp SDT and fuzzy SDT when dealing with sensitivity and bias; experimentation with different permutations and combinations of mapping ' $\mathrm{s}$ ' and ' $\mathrm{r}$ ' values (for example, fuzzy SDT analysis can be extended to cases in which the signal is fuzzy, but the response is discrete or binary); experimentation with population from other trades and other geographic regions thereby yielding a much larger and richer data set; designing a survey for the officials who define safety standards, which would help get information as to how well they are aware of industry practices onsite.

\section{CONCLUSION}

Safety training is only as good as the worker's ability to detect an unsafe condition when he/she is presented with one. Crisp SDT as implemented by [1] and [3] provided a good tool for an assessment of this ability. However, the assumptions of the conventional SDT model may not be appropriate for construction-specific situations.

Preliminary results presented in this study indicate that fuzzy SDT captures useful information that would have otherwise been lost with crisp SDT. It appears that fuzzy SDT provides a finer resolution of a worker's ability in identifying hazardous conditions on a construction jobsite. Overall, this model could be used in any real world setting that involves assessment of a decision making process.

Crisp SDT had been applied earlier to assessment of workers' occupational safety competencies. This research further strengthens the analysis by proposing the use of fuzzy SDT. SDT in its original form is as robust a theory as any other. Fuzzy SDT only improves its application. The group of ironworkers surveyed was found to have an above average sensitivity, i.e., they were able to differentiate and detect an unsafe condition from a safe one. The group had a conservative strategy, whereby; they would even consider some of the safe conditions as unsafe, yielding a high false alarm rate. The outcome of the survey could have been very different, based on the sample of ironworkers selected or if the survey questions were altered.
The intent of this research was to demonstrate an accurate and befitting assessment of the workers ability to identify a hazard. The survey questionnaire provides a solid foundation for next steps to develop valuable, reliable tools for assessment of safety competency. With further development and testing, it can be used in current safety training in the construction industry.

\section{REFERENCES}

[1] Formoso C T, Ed. Signal detection theory: Enabling work near the edge: Proceedings of the $11^{\text {th }}$ Annual Conference for Lean Construction; Virginia Tech, 2003.

[2] Formoso C T, Ballard G, Eds. Signal detection theory: Working near the edge: a new approach to construction safety: Proceedings of the $10^{\text {th }}$ Annual Conference for Lean Construction; 2000, NORIE/UFRGS.

[3] Patel BJ. Assessment of construction workers occupational safety competencies using signal detection theory. Masters [dissertation]. East Lansing (MI): Michigan State University; 2003.

[4] Parasuraman R, Masalonis AJ. Fuzzy signal detection theory: Basic postulates and formulas for analyzing human and machine performance. Hum Factors 2000; 42: 636-59.

[5] Hinze JW, Ed. Construction safety. Upper Saddle River, New Jersey: Prentice Hall 1997.

[6] Vargas CA. Investigating construction falls using fault tree analysis and developing a prototype tool to reduce falls using expert system and computer assisted instruction method. PhD. [dissertation]. Columbus (OH): The Ohio State University; 1998.

[7] McCann M. Cause of roofer death. The Center to Protect Workers' Rights 2000.

[8] Hinze J, Wilson G. Moving toward a zero injury objective. J Constr Engrg Mgmt 2000; 126: 399-403.

[9] Pollack E, Chowdhury R. Trends in work-related death and injury rates among U.S construction workers, 1992-98. The Center to Protect Workers' Rights 2001.

[10] Mohamed S. Safety climate in construction site environments. J Constr Engrg Mgmt ASCE 2002; 128: 375-84.

[11] Fredericks T, Abduayyeh O, Palmquist M, Torres HN. Mechanical contracting safety issues. J Constr Engrg Mgmt 2002; 128: 186-93.

[12] Gambatese JA. Safety in a designer's hands. Civ Engrg ASCE 2000; 20: 56-9.

[13] Hinze J, Wiegand F. The role of designers in construction worker safety. J Constr Engrg Mgmt ASCE 1992; 118: 677-84.

[14] Suraji A, Duff AR, Peckitt SJ. Development of causal model of construction accident causation. J Constr Engrg Mgmt ASCE 2001; 127: 337-44.

[15] McClay RE. Toward a more universal model of loss incident causation-part I. Professional Safety 1989.

[16] McClay RE. Toward a more universal model of loss incident causation-part II. Professional Safety 1989; pp. 34-9.

[17] Hinze J, Ed. The distraction theory of accident causation. Proc. International Conference on implementation of safety and health on construction sites, CIB Working Commission W99: Safety and Health on Construction Sites: 1996, Balkema, Rotterdam, The Netherlands; Alvez LM, Diaz RJ, Coble, Eds: 1996; pp. 357-84.

[18] Abdelhamid TS, Everett JG. Identifying root causes of construction accidents. J Constr Engrg Mgmt ASCE 2000; 126: 52-60.

[19] Toole TM. Construction site safety roles. J Constr Engrg Mgmt ASCE 2002; 128: 203-10.

[20] Gibb A, Hide S, Haslam R, et al. Construction tools and equipment - their influence on accident causality. J Engrg Des Tech 2005; 3 : 12-23.

[21] Rasmussen J, Pejtersen AM, Goodstein LP, Eds. Cognitive system engineering. New York: John Wiley and Sons, Inc. 1994.

[22] Rasmussen J. Risk management in a dynamic society: A modeling problem. Safety Sci 1997; 27: 2/3, 183-213.

[23] Swets JA, Ed. Signal detection theory and ROC analysis in psychology and diagnostics: collected papers. Mahwah: Lawrence Erlbaum Associates 1996.

[24] Heeger D, Ed. Writings on signal detection theory (Advanced). Professor of Psychology and Neural Science, New York University 1997. 
[25] Masalonis AJ, Parasuraman R. Fuzzy signal detection theory: Analysis of human and machine performance in air traffic control, and analytic considerations. Ergonomics 2003; 46(11): 1045-74.

[26] Narang P. Analysis of construction safety competencies using fuzzy signal detection theory. Masters [dissertation]. E. Lansing (MI): Michigan State University 2005.

(C) Adbelhamid et al.; Licensee Bentham Open.

This is an open access article licensed under the terms of the Creative Commons Attribution Non-Commercial License (http://creativecommons.org/licenses/by-nc/3.0/) which permits unrestricted, non-commercial use, distribution and reproduction in any medium, provided the work is properly cited. 